\title{
Toxicological studies on commercial hair dye brand commonly used in Saudi Arabia: histological study of the liver, kidney, heart, and testis
}

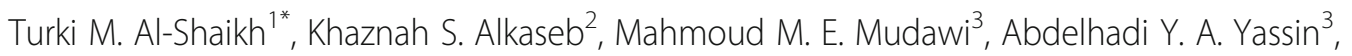
Rami S. Habeballa ${ }^{3}$ and Shiemaa D. Alzain ${ }^{3}$

\begin{abstract}
Background: Hair dye is commonly used in the Kingdom of Saudi Arabia (KSA). Poisoning occurs when someone swallows dye or tint used to color hair and is considered one of the most significant causes of intentional self-harm. Commercial hair dye may contain varying amounts of paraphenylenediamine and even undeclared chemical ingredients. Therefore, the aim of this study was to evaluate the organ toxicity of commercial hair dye in rats.

Acute toxicity study: The commercial hair dye X was orally administered to the rats at a dose of $50 \mathrm{mg} / \mathrm{kg}$ and was observed for $24 \mathrm{~h}$ for mortality or behavioral changes.

Sub-chronic toxicity: The commercial hair dye X was administered to rats orally for 8 days in doses of 2.5 and $5 \mathrm{mg} / \mathrm{kg}$, then the heart, liver, kidney, and testis were dissected out of the animals and kept in formalin for histopathological examinations using $\mathrm{H}$-E stain.

Results: In this study, the commercial hair dye $X$ at the dose of $50 \mathrm{mg} / \mathrm{kg}$ single dose has not shown any mortality in rats. On the other hand, the commercial hair dye $X$ administered to rats orally for 8 days at doses of 2.5 and $5 \mathrm{mg} / \mathrm{kg}$ caused toxicological effects manifested by some histopathological changes in the heart, liver, kidney, and testis in rats compared to the control group.

Conclusion: The current study showed that the commercial hair dye $X$ at doses of 2.5 and $5 \mathrm{mg} / \mathrm{kg}$ produced toxic effects on the heart, liver, kidney, and testis in rats. Therefore, it is important to raise the public awareness about the potential toxicity of the hair dyes.
\end{abstract}

Keywords: Hair dye, Hair dye poisoning, Paraphenylenediamine, Histopathology

\section{Background}

The art of hair dyeing was known as early as 5000 years BC among the Egyptians. Historically, hair has functioned as a social indicator of attractiveness, femininity, masculinity, health, and beauty (Saitta et al., 2013; Zaid, Al-ramahi, Abu ghoush, Jaradat, \& Musmar, 2013).

In a cross-sectional survey conducted in Saudi Arabia about hair dye, among respondents, $82.6 \%(464 / 562)$ had at some point dyed their hair. Furthermore, 69.3\% (334/

\footnotetext{
* Correspondence: turkialsheika@hotmail.com

'Department of Biological Sciences, Faculty of Science, Northern Border

University, Arar, Saudi Arabia

Full list of author information is available at the end of the article
}

482) had dyed their hair in the past 12 months and 76 . $8 \%(354 / 461)$ of the participants used permanent dyes (Alghamdi \& Moussa, 2012).

Poisoning with hair dye containing paraphenylenediamine is a new trend of intentional self-harm in various developing countries, and consumption of hair dye as a deliberate means of self-harm has been reported from different regions in India, Asia, and Africa, among adult patients and Sudanese children (Chandran, Manners, Agarwal, \& Ebenezer, 2012; Sakuntala et al., 2015; Senthilkumaran \& Thirumalaikolundusubramania, 2015).

The major ingredient in most hair dyes is paraphenylenediamine (PPD) in concentration ranging from 2 to $10 \%$ 
(Senthilkumaran \& Thirumalaikolundusubramania, 2015). The effects of PPD when ingested are serious and may cause cervicofacial edema, mucosal injury, respiratory distress, acute renal failure, rhabdomyolysis, and myocardial injury, cardiotoxicity and arrhythmias as well as atrophy of the optic nerve resulting in blindness in a patient who ingested an estimated amount of $7 \mathrm{~g}$ paraphenylenediamine and cause death (Bhargava \& Matthew, 2007; Chandran et al., 2012; Kondle, Pathapati, Saginela, Malliboina, \& Makineedi, 2012; Nohyneka, Fautzb, BenechKiefferc, \& Toutaina, 2004; Sanchez, Handyal, Kannan, Siddalingeshwara, Alvarez-Uria, Gavalda, \& Cobella, 2016; Senthilkumaran \& Thirumalaikolundusubramania, 2015). It is reported that some commercial hair dyes contain a potent nephrotoxic cocktail containing PPD, propylene glycol, and resorcinol and histological changes of acute tubular necrosis have been described in PPD poisoning (Prabhakar \& Kamalakar, 2012). The characteristic chocolate brown color of urine could be confirmative evidence of hair dye poisoning in an individual with the poisoning of PPD. This later is thought to have abortive effect in rural Africa (Zaid, Al-ramahi, Abu ghoush, Jaradat, \& Musmar, 2013). There is no antidote for PPD poisoning (Prabhakar \& Kamalakar, 2012; Sakuntala et al., 2015). If the poisoning is not recognized early, it has a very high incidence of mortality (Garg, Tiwari, \& Ahlawat, 2014). The edema of the face, neck, and laryngeal region could be severe enough to cause respiratory distress and hypoxia, and necessitate an early and emergency intubation or tracheostomy which is lifesaving in such patients (Chandran et al., 2012).

As poisoning due to PPD has a high mortality incidence, therefore, early recognition can be lifesaving. As there is no specific antidote, the management of poisoning includes gastric lavage, antihistamines, parenteral steroids, and alkalinization of the urine. Respiratory distress is the major early challenge, which may require ventilator support (Kondle et al., 2012). Renal support in the form of dialysis is required in acute renal failure (Kondle et al., 2012). Limited data were available on the head to head comparison of hydrocortisone and methyl prednisolone in hair dye poisoning (Kondle et al., 2012).

The concentration of PPD in hair dye formulation varies from 70 to $90 \%$ in stone hair dye and $2-10 \%$ in branded dyes (Kondle et al., 2012), which are used for giving black color to hair. Exact concentrations of PPD in different formulations are not known because most hair dye formulations are proprietary. PPD is rapidly absorbed into the blood through mucous membranes of the digestive tract after its oral intake (Senthilkumaran \& Thirumalaikolundusubramania, 2015).

The results of acute dermal toxicity studies suggest that hair dye ingredients are non-toxic via the topical route (Nohyneka et al., 2004). With the exception of allergy or rare cases of irritation due to the presence of alkaline additives, hair dye products have an adequate local tolerance under standard conditions of use (Nohyneka et al., 2004). In susceptible individuals, PPD usually can cause contact dermatitis (Punjan, 2014). However, the major systemic problem occurs when it is ingested accidentally, for purposes of suicidal intent or during attempted murder. If ingested, PPD can cause angioedema of the neck and face, acute renal failure, and rhabdomyolyses (Punjan, 2014).

Hair dye X (the brand hair dye selected for the current study): $\mathrm{X}$ hair dye is a commercial hair dye brand commonly used in KSA and will be used for the current study. According to the components of hair dye $\mathrm{X}$, it contains a lot of chemicals that may have serious side effects. The listed ingredients in the hair dye X brochure-in orderare P-phenylenediamine sulfate, sodium perborate, cellulose gum, m-aminophenol, sodium carbonate, disodium lauryl sulfosuccinate, magnesium stearate, p-aminophenol, fragrance (perfume), and algin, sapindus mukurossi peel extract.

\section{Rationale of the study}

Hair dye is commonly used and poisoning occurs when someone swallows dye or tint used to color hair and is considered one of the most significant causes of intentional self-harm; hair dye poisoning also can occur unintentionally like while preparing the dye or it can be absorbed to the blood through the skin. Since the commercial hair dye may contain varying amounts of paraphenylenediamine and even undeclared chemical ingredients; therefore, the aim of this study was to evaluate the toxicity of commercial hair dye used in Saudi Arabia in rats.

\section{Methods \\ Hair dye}

The most widely used commercial brands of hair dye in Saudi Arabia (KSA) were determined and one is selected and purchased from the local market and given the name $\mathrm{X}$ for the current study.

\section{Animals}

Male Wistar rats weighing 150-200 g were used in this study. The animals were kept and maintained under laboratory conditions in Northern Border University, Kingdom of Saudi Arabia, and were allowed free access to food (standard pellet diet) and water ad libitum. The animals were divided into three groups of five animals per each.

\section{Methods}

\section{Acute toxicity study}

Acute toxicity study for the commercial hair dye $\mathrm{X}$ was done according to the OECD guidelines no: 423 (2001). The commercial hair dye $\mathrm{X}$ was orally administered to 
the rats $(n=3)$ at dose of $50 \mathrm{mg} / \mathrm{kg}$ and observed for $24 \mathrm{~h}$ for mortalities or behavioral changes.

\section{Sub-chronic toxicity}

The animals were divided into three experimental groups (five rats for each group) as follows:

1. Group I: normal control received distilled water orally

2. Group II: received $2.5 \mathrm{mg} / \mathrm{kg}$ hair dye orally

3. Group III: received $5 \mathrm{mg} / \mathrm{kg}$ hair dye orally

The animals administered with the respective treatment for 8 days and then the heart, liver, kidney, and testis were dissected and fixed in formalin for histopathological examinations using H-E stain (Al-Shaikh, 2016).

\section{Results}

Acute toxicity

As shown in Table 1, the commercial hair dye $\mathrm{X}$ at the dose of $50 \mathrm{mg} / \mathrm{kg}$ administered orally has not shown any significant toxic effects in rats.

\section{Sub-chronic toxicity}

\section{Heart}

Sections from the rat cardiac muscle (left ventricle) indicates the normal control group (G1: Co) with normal cardiac muscle fibers having oval central nuclei and thin wall intervening capillaries $(\mathrm{H}-\mathrm{E}, \times 200)$; While the group received the low dose (G2: LD) showing focal atrophy with mononuclear inflammatory cells and numerous dilated and congested vessels $(\mathrm{H}-\mathrm{E}, \times 200)$ and the group received the high-dose (G3: HD) dye showed marked disorganization and damage of cardiac fibers with focal regions of severe necrosis and cardiac fiber loss $(\mathrm{H}-\mathrm{E}, \times 200)$ Fig. 1 .

\section{Liver}

Central vein region Sections in the rat liver at central vein region $(\mathrm{CV})$ show the control group (G1: $\mathrm{CO})$ with normal hepatocyte cell cords and thin intervening sinusoids $(\mathrm{H}-\mathrm{E}, \times 200)$. While the low-dose group (G2: LD) showing mild granularity of hepatocytes and nuclei with dark stained dense chromatin, sinusoidal congestion, and focal hemorrhage. Central vein $(\mathrm{CV})$ showed endothelial lining damage $(\mathrm{H}-\mathrm{E}, \times 200)$, and the group administered with the high-dose dye (G3: HD) showed marked central vein $(\mathrm{CV})$ congestion and vacuolar degeneration of nearby hepatocytes. Others on the right are dark stained shrunken and degenerated $(\mathrm{H}-\mathrm{E}, \times 200)$ Fig. 2.

Liver (portal) As shown in the sections from the rat liver at portal regions, the group received low-dose dye (G2: LD) showing hepatocytes with dark small degenerated nuclei, congested portal vein (PV), and bile duct (BD) surrounded by inflammatory cells (H-E, × 200), while the group administered with the high-dose dye (G3: HD) showed congested portal veins (PV) and marked hydropic degeneration of hepatocytes where cells are swollen with unstained cytoplasm and small dark nuclei compressing sinusoids (hardly identified) (HE, $\times 200)$ Fig. 3 .

\section{Kidney}

Sections from the rat kidney show the renal tubules of the following: G1: Co (control) group, proximal tubules showed intact pyramidal cells surrounded narrow lumen. The cytoplasm is highly acidophilic, and nuclei are rounded and vesicular (active) $(\mathrm{H}-\mathrm{E}, \times 400)$. G2-a: LD dye (low dose) showing slight changes in proximal tubules in the form of increase cytoplasmic acidophilia and density of nuclei staining (signs of early apoptosis). Distal tubules showed dilated lumina with decrease height of lining epithelium (H-E, $\times 400)$. G2-b: LD dye showed loss of tubular outlines. The lumina were obliterated. The cytoplasm of cells is deeply stained and the nuclei become smaller and deeply stained (signs of apoptosis) $(\mathrm{H}-\mathrm{E}, \times 400)$.

G3: HD dye (high dose) showing disorganization of tubular shape, dilatation and signs of cell degeneration (unstained cytoplasm with decrease nuclear size (dark stained) $(H-E, \times 400)$ Fig. 4.

\section{Testis}

Sections from the rat testis G1 (control group) showing seminiferous tubules (ST) interstitial Leydig cells were seen among the tubules $(\mathrm{H}-\mathrm{E}, \times 200)$ Fig. 5.

Table 1 The acute toxicity test of the commercial hair dye $X$ at a dose of $50 \mathrm{mg} / \mathrm{kg}$

\begin{tabular}{|c|c|c|c|c|c|c|c|c|c|c|c|c|c|c|c|c|c|}
\hline \multirow[t]{3}{*}{ Rat no. } & \multirow{3}{*}{$\begin{array}{l}\text { Dose } \\
(\mathrm{mg} / \mathrm{kg})\end{array}$} & \multicolumn{14}{|c|}{ Physiological alterations (h) } & \multirow{2}{*}{\multicolumn{2}{|c|}{ Mortality (h) }} \\
\hline & & \multicolumn{2}{|c|}{ Grooming } & \multicolumn{2}{|c|}{ Hyperactivity } & \multicolumn{2}{|c|}{ Sedation } & \multicolumn{2}{|c|}{ Resp. arrest } & \multicolumn{2}{|c|}{ Convulsions } & \multicolumn{2}{|c|}{$\begin{array}{l}\text { Increased motor } \\
\text { activity }\end{array}$} & \multicolumn{2}{|c|}{$\begin{array}{l}\text { Decreased motor } \\
\text { activity }\end{array}$} & & \\
\hline & & $30 \mathrm{~min}$ & $24 \mathrm{~h}$ & $30 \mathrm{~min}$ & $24 \mathrm{~h}$ & $30 \mathrm{~min}$ & $24 \mathrm{~h}$ & $30 \mathrm{~min}$ & $24 \mathrm{~h}$ & $30 \mathrm{~min}$ & $24 \mathrm{~h}$ & $30 \mathrm{~min}$ & $24 \mathrm{~h}$ & $30 \mathrm{~min}$ & $24 \mathrm{~h}$ & $30 \mathrm{~min}$ & $24 \mathrm{~h}$ \\
\hline 1 & 50 & - & - & - & - & - & - & - & - & - & - & - & - & - & - & - & - \\
\hline 2 & 50 & - & - & - & - & - & - & - & - & - & - & - & - & - & - & - & - \\
\hline 3 & 50 & - & - & - & - & - & - & - & - & - & - & - & - & - & - & - & - \\
\hline
\end{tabular}



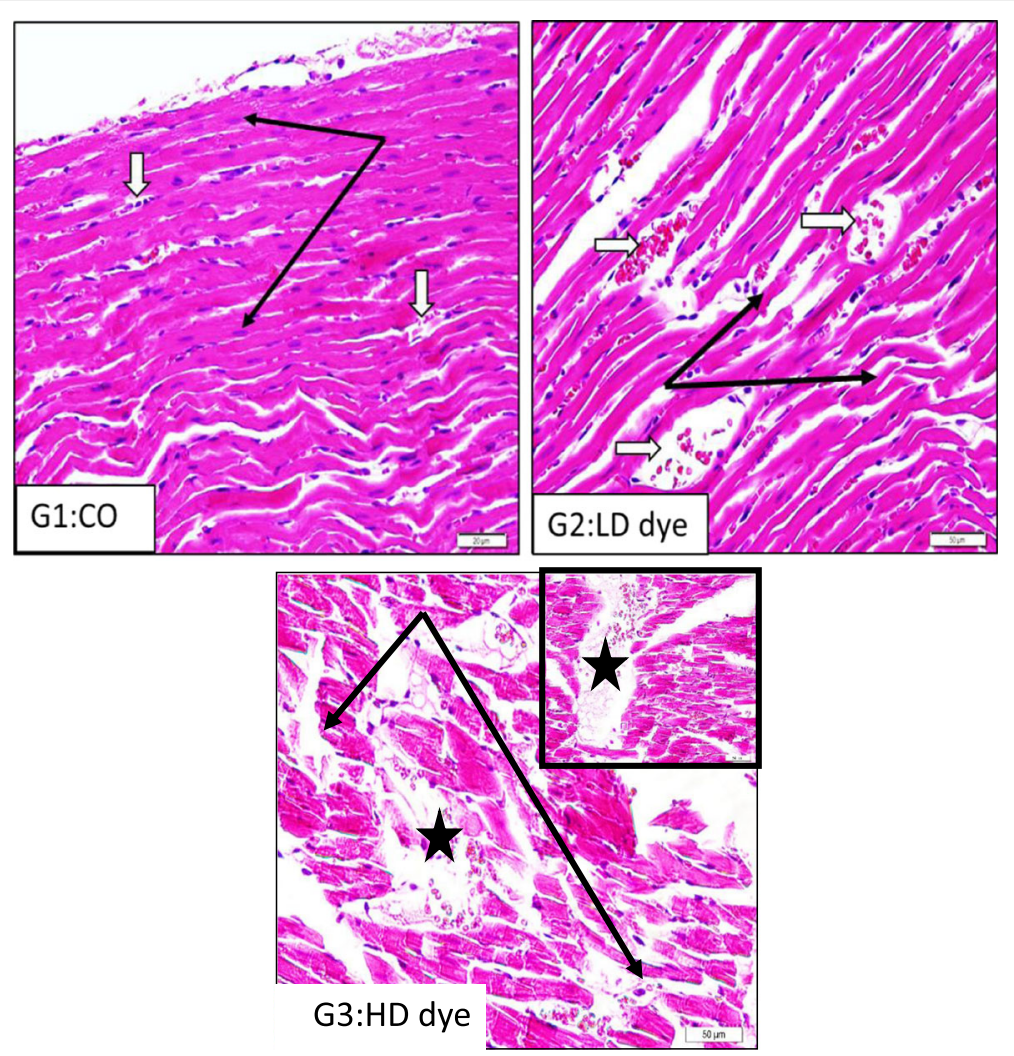

Fig. 1 Sections in the rat cardiac muscle (left ventricle) show the following: G1: Co (control) with normal cardiac muscle fibers having oval central nuclei (black arrows) and thin wall intervening capillaries (white arrows) $(\mathrm{H}-\mathrm{E}, \times 200)$. G2: LD dye (low dose) showing focal atrophy with mononuclear inflammatory cells (black arrows) and numerous dilated and congested vessels (white arrows) (H-E, $\times 200)$. G3: HD dye (high-dose dye) showing marked disorganization and damage of cardiac fibers (arrows) with focal regions of severe necrosis fiber loss (stars) $(H-E, \times 200)$

Testis (low dose) Sections from the rat testis administered with low-dose hair dye for 8 days showing the following: (a) deformity and shrinkage of seminiferous tubules. Interstitial spaces were increased with compensatory hyperplasia of Lyedig cells $(\mathrm{H}-\mathrm{E}, \times 200)$. (b) Higher magnification to show deformed tubule with homogenous material within the lumen masking the few number of mature sperms and increase or hyperplasia of Lyedig cells. Increase tissue spaces due to atrophy of tubules (notice the blood vessel with thick wall) (H-E, $\times$ 200). (c) Some samples showed basal vacuoles between germ cells. Tissue spaces are also widened with hyperplasia of Leydig cells $(\mathrm{H}-\mathrm{E}, \times 200)$ Fig. 6 .

Testis (high dose) Sections from the rat testis administered with high-dose hair dye for 8 days show the following: (a) more shrinkage and deformity of seminiferous tubule, wide interstitial spaces, and dilatation and congestion of
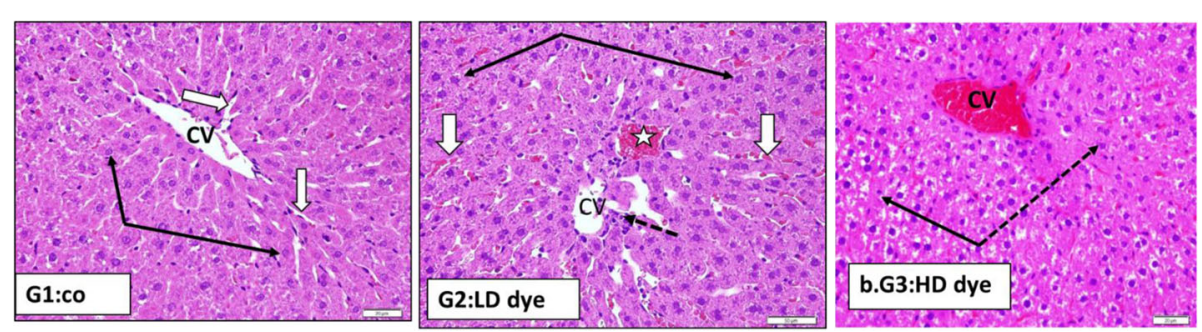

Fig. 2 Sections in the rat liver central vein region (CV) show the following: G1: CO (control) group with normal hepatocyte cell cords (black arrows) and thin intervening sinusoids (white arrows) $(H-E, \times 200)$. G2: LD dye (low dose) showing mild granularity of hepatocytes and nuclei with dark stained dense chromatin (black arrows), sinusoidal congestion (white arrows), and focal hemorrhage (star). Central vein (CV) showed endothelial lining damage (dotted arrow) $(\mathrm{H}-\mathrm{E}, \times 200)$. G3: HD dye (high dose) with marked central vein (CV) congestion and vacuolar degeneration of nearby hepatocytes (arrows), swollen sinusoids that could not be seen. Others on the right are darkly stained shrunken and degenerated (dotted arrows) $(\mathrm{H}-\mathrm{E}, \times 200)$ 


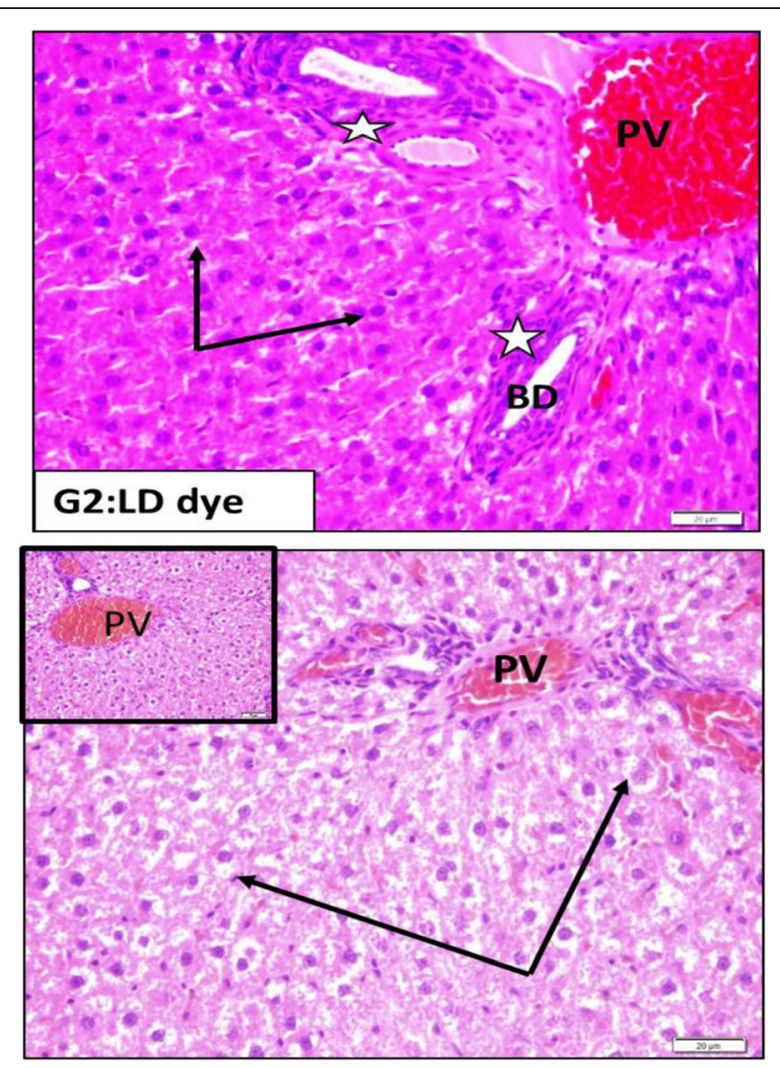

Fig. 3 Sections from the rat liver at portal regions show the following: G2: LD dye (low dose) showing hepatocytes with dark small degenerated nuclei (arrows), congested portal vein (PV), and bile duct $(B D)$ surrounded by inflammatory cells (stars) $(H-E, \times 200)$

blood vessels $(\mathrm{H}-\mathrm{E}, \times 200)$. (b) Magnified part shows degenerative changes in basal cells. The nuclei of other germ cells are dark stained and pyknotic marked congestion of blood vessels (BV) with perivascular inflammatory cells and atrophy and degeneration of Leydig cells $(\mathrm{H}-\mathrm{E}, \times 400)$. (c) Some tubules showed marked degeneration and absence of most germ cells; the rest showed no mature sperms. Leydig cells looked small and degenerated $(\mathrm{H}-\mathrm{E}, \times 400)$ Fig. 7 .

\section{Discussion}

Historically, hair painting has functioned as a social indicator of attractiveness, femininity, masculinity, health, and beauty (Saitta et al., 2013; Zaid et al., 2013). Many cases of toxicity and mortality either due to accidental or deliberate ingestion of hair dyes were reported (Abdel-Moneim, 2017; Tiwari, Jatav, \& Dudani, 2016).

The main component of hair dye causing toxicity is paraphenylenediamine (PPD). This compound has been found to cause angioneurotic edema, rhabdomyolysis, and renal failure (Abdelraheem et al., 2010; Bhargava \& Matthew, 2007; Punjan, 2014). Severe edema of the face, neck, and floor of the mouth, renal failure, and myocarditis were poor prognostic factors (Jain et al., 2011). Almost all body systems are affected with varying degrees (Hassan ARahman \& Ahmed, 2014).

The compound PPD is highly toxic. When taken orally, death occurs within the first 6-24 h due to angioneurotic edema. Smaller doses cause angioneurotic edema and hepatitis while moderate doses cause acute renal failure within the first week. Despite the high frequency of cases and mortality, no antidote is available for this poisoning (Sakuntala et al., 2015).

Resorcinol, propylene glycol, liquid paraffin, cetostearyl alcohol, sodium lauryl sulfate, ethylenediaminetetraacetic acid (EDTA) sodium, herbal extracts, preservative, and perfume are other ingredients present in small amounts in commercial hair dye (ChuaGocheco, Bozzo, \& Einarson, 2008). Resorcinol is a corrosive, acid, and protein denaturant causing severe burns on direct contact. Toxic effects of these ingredients could have had additive effect in the patient (Chandran et al., 2012). The combined effect of the individual compounds may be responsible for its significant morbidity and mortality (Kondle et al., 2012).

It is reported that PPD intoxication cause systemic inflammatory reaction specific to a cytotoxic cell support. The pathophysiologic mechanisms could be due to increased free radical formation, skeletal and cardiac muscle necrosis (scattered coagulation necrosis), formation of highly nephrotoxic quinonediamine (an oxidation product of PPD metabolites), renal tubular occlusion due to myoglobin casts, and acute tubular necrosis (Senthilkumaran \& Thirumalaikolundusubramania, 2015).

In this research, acute toxicity study was performed in rats to assess the toxic effect of the commercial hair dye commonly used in Saudi Arabia; it was found that the hair dye $X$ at the dose of $50 \mathrm{mg} / \mathrm{kg}$ orally did not show any significant toxic effects in rats. No death and no behavioral changes were observed within $24 \mathrm{~h}$. This agrees with other studies which reported that hair dyes and their ingredients have moderate to low acute toxicity (Nohyneka et al., 2004).

In this study, the commercial hair dye $\mathrm{X}$ was orally administered to rats for 8 days at doses of 2.5 and $5 \mathrm{mg} / \mathrm{kg}$ to study the histopathological effects on the heart, liver, kidney, and testis.

It is reported that the most common histopathological changes in all studied organs were vascular congestion and lymphocyte infiltration, with degenerative changes in the hepatocytes and the destruction of the renal tubules (Abdel-Moneim, 2017).

In this study, the effect of low dose of hair dye $\mathrm{X}$ on the rat heart showed focal atrophy with mononuclear inflammatory cells, numerous dilated and congested vessels while the high dose showed marked disorganization and damage of cardiac fibers with focal regions of severe necrosis and fiber loss. 

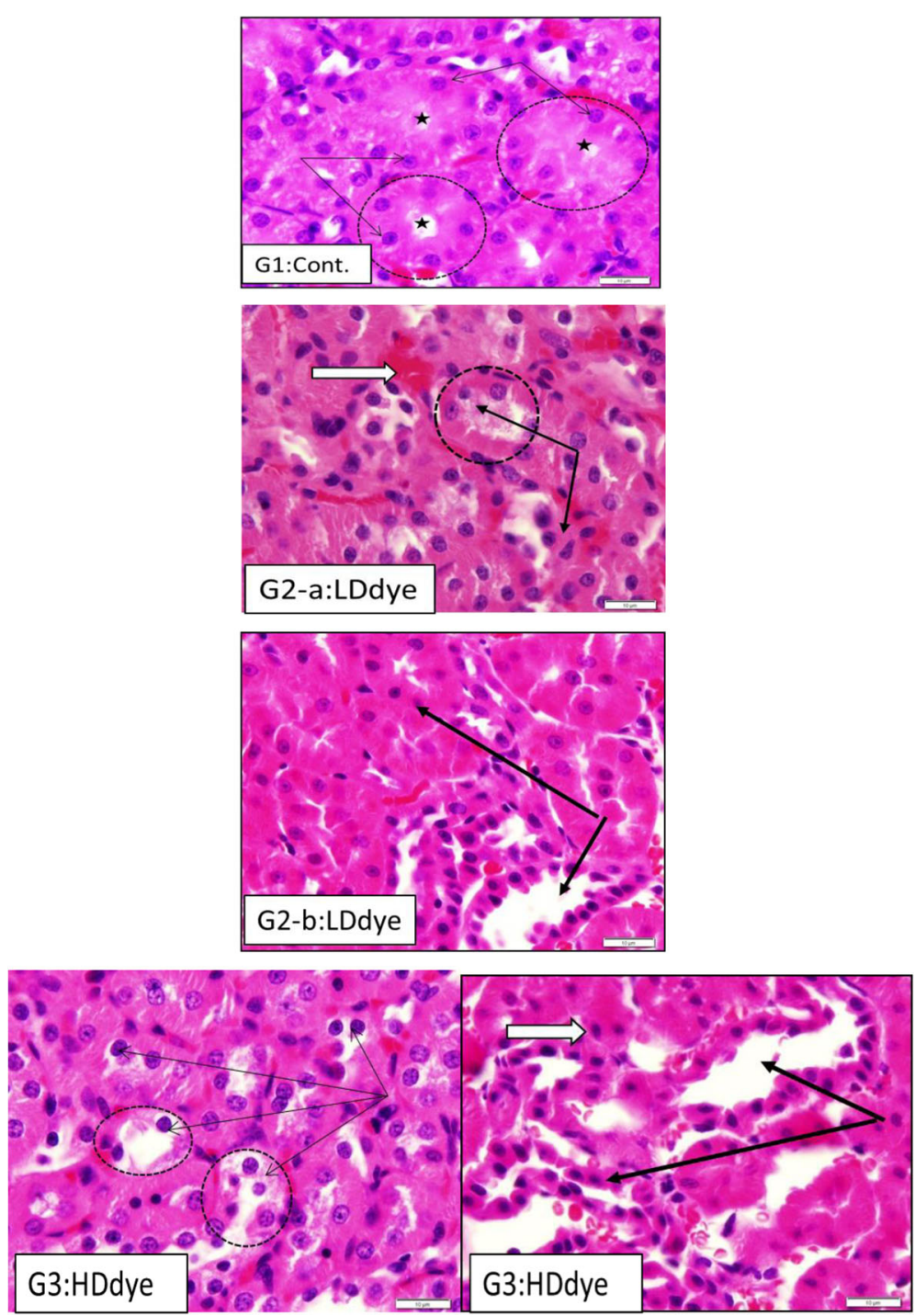

Fig. 4 Sections from the rat kidney show renal tubules of the following: G1: Co (control) group, proximal tubules (dotted circles) showing intact pyramidal cells surrounded by narrow lumen (stars), the cytoplasm highly acidophilic and rounded, vesicular (active) nuclei (arrows) are $H-E, \times$ 400. G2: LD dye (low dose) showing slight changes in proximal tubules in the form of increase cytoplasmic acidophilia and density of nuclei staining (signs of early apoptosis). Distal tubules showed dilated lumina with decrease height of lining epithelium. Some tubular cells showed unstained cytoplasm $(H-E, \times 400)$. G2-a: LD dye (low dose) showing slight changes in proximal tubules in the form of increase cytoplasmic acidophilia and density of nuclei staining (signs of early apoptosis). Distal tubules showed dilated lumina with decrease height of lining epithelium. G2-b: LD dye showed loss of tubular outlines. The lumina were obliterated. The cytoplasm of cells is deeply stained, and the nuclei become smaller and deeply stained (signs of apoptosis). (H-E, $\times 400)$. G3: HD dye (high dose) showing disorganization of tubular shape, dilation (arrows), and signs of cell degeneration (unstained cytoplasm (dotted circles) with decrease nuclear size (dark stained) (white arrows) $(H-E, \times 400)$

Several studies reported that myocardial damage and myocarditis are less frequently in hair dye poisoning but associated with higher mortality (Chandran et al., 2012; Hassan A-Rahman \& Ahmed, 2014).

Myocarditis is a fatal and a commonly neglected complication of PPD poisoning. However, oral ingestion of PPD in doses $>10 \mathrm{~g}$ especially unbranded stone hair dyes results in extensive myocardial damage (Senthilkumaran \& Thirumalaikolundusubramania, 2015; Tiwari et al., 2016).
Cardiac toxicity is mainly caused by the direct toxic effect of PPD on the heart and rhabdomyolysis of the cardiac muscle causing severe damage and hyperkalemia (Tiwari et al., 2016).

In the current study, the hair dye X administered in low dose produced histopathological changes in the rat liver. Central veins of hepatic sections from this group looked deformed with degenerated endothelial lining; hepatocytes looked enlarged or swollen with increase acidophilic staining of cytoplasm (granular appearance) 


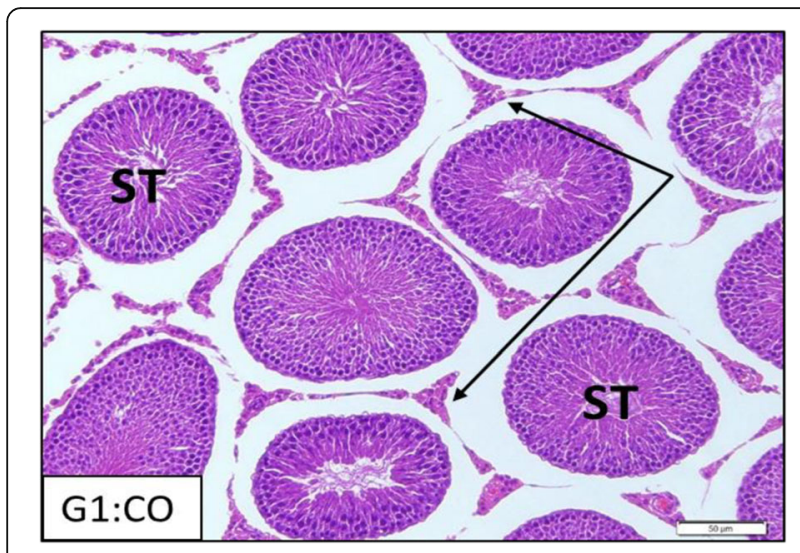

Fig. 5 Control group: sections from the rat testis stained showing seminiferous tubules (ST) interstitial Leydig cells were seen among the tubules (black arrows) $(H-E, \times 200)$

that may be related to proliferation of mitochondria and smooth endoplasmic reticulum for detoxification. Nuclei also looked darker with increased density of chromatin compared to control. There was focal congestion of blood sinusoids. The effect of low dose of hair dye $\mathrm{X}$ in the portal region indicated hepatocytes with dark small degenerated nuclei, congested portal vein (PV), and bile duct (BD) surrounded by inflammatory cells.

The high dose of hair dye $\mathrm{X}$ produced more changes in the central vein region in the form of central vein congestion (CV); hepatocytes are more swollen compressing sinusoids. The cytoplasm of cells showed unstained regions around the nuclei indicating degenerative changes of organelles. The nuclei looked smaller and darker (signs of pyknosis). The effect of high dose in the portal region showed congested portal veins (PV) and marked hydropic degeneration of hepatocytes where cells are swollen with unstained cytoplasm and small dark nuclei compressing sinusoids.

Many studies documented the toxic effects of hair dye on the liver. Consumption of even lower volumes resulted in hepatitis (Kondle et al., 2012). Tender palpable liver with features of acute hepatitis on liver biopsy was seen in many patients (Abdelraheem et al., 2010). In a study which analyzed 25 hair dye poisoning deaths, the histological examination of liver cross section showed focal areas of fatty change and central vein dilatation in $48 \%$ of the cases due to systemic toxicity (Alugonda, Maddileti, Naga Lingam, \& Rangaiah, 2013).

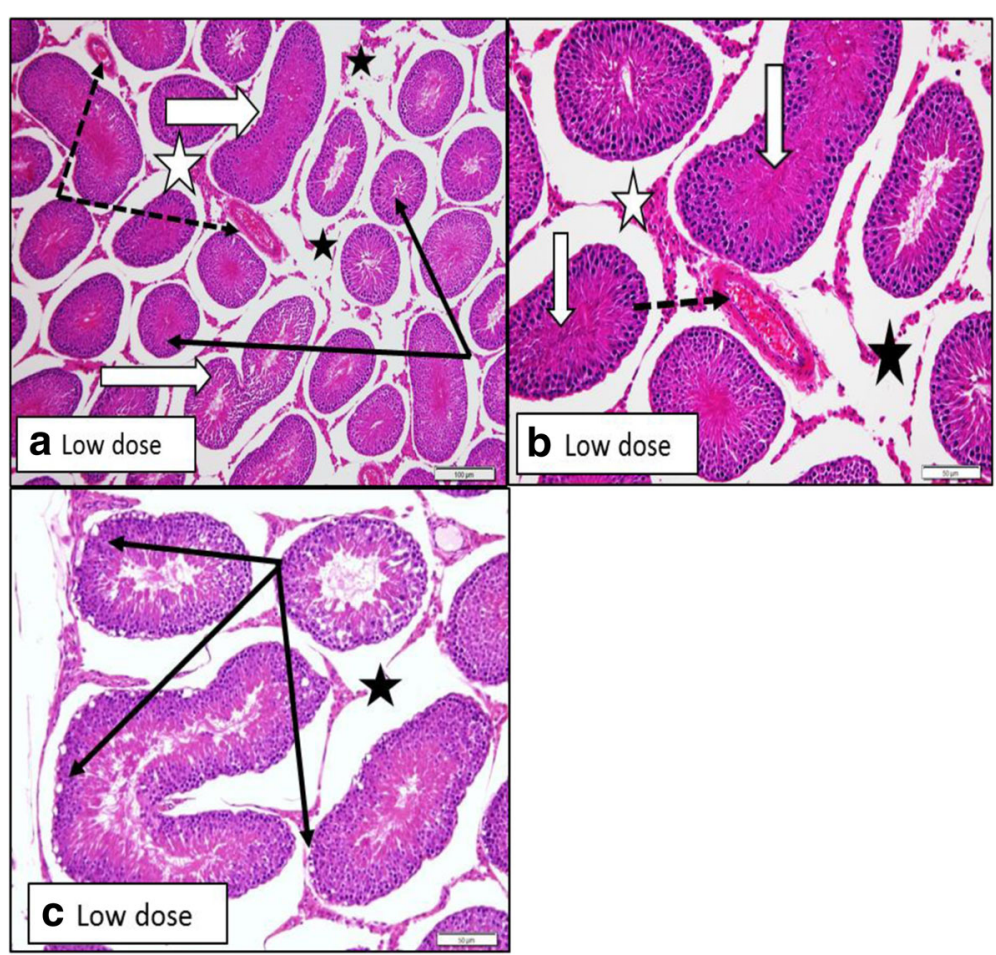

Fig. 6 Sections from the rat testis administered with low-dose hair dye for 8 days show the following: a deformity (white arrows) and shrinkage (thin black arrows) of seminiferous tubules. Interstitial spaces were increased (stars) with compensatory hyperplasia of Lyedig cells (white star) (H-E, $\times 200)$. $\mathbf{b}$ Higher magnification show deformed tubule with homogenous material within the lumen masking the few number of mature sperms (white arrows), increase or hyperplasia of Lyedig cells (white star), and increase of tissue spaces due to atrophy of tubules (black star) (notice the blood vessel with thick wall) (dotted arrow) $(H-E, \times 200)$. c Some samples showed basal vacuoles between germ cells (black arrows). Tissue spaces are also widen (black stars) with hyperplasia of Leydig cells $(H-E, \times 200)$ 


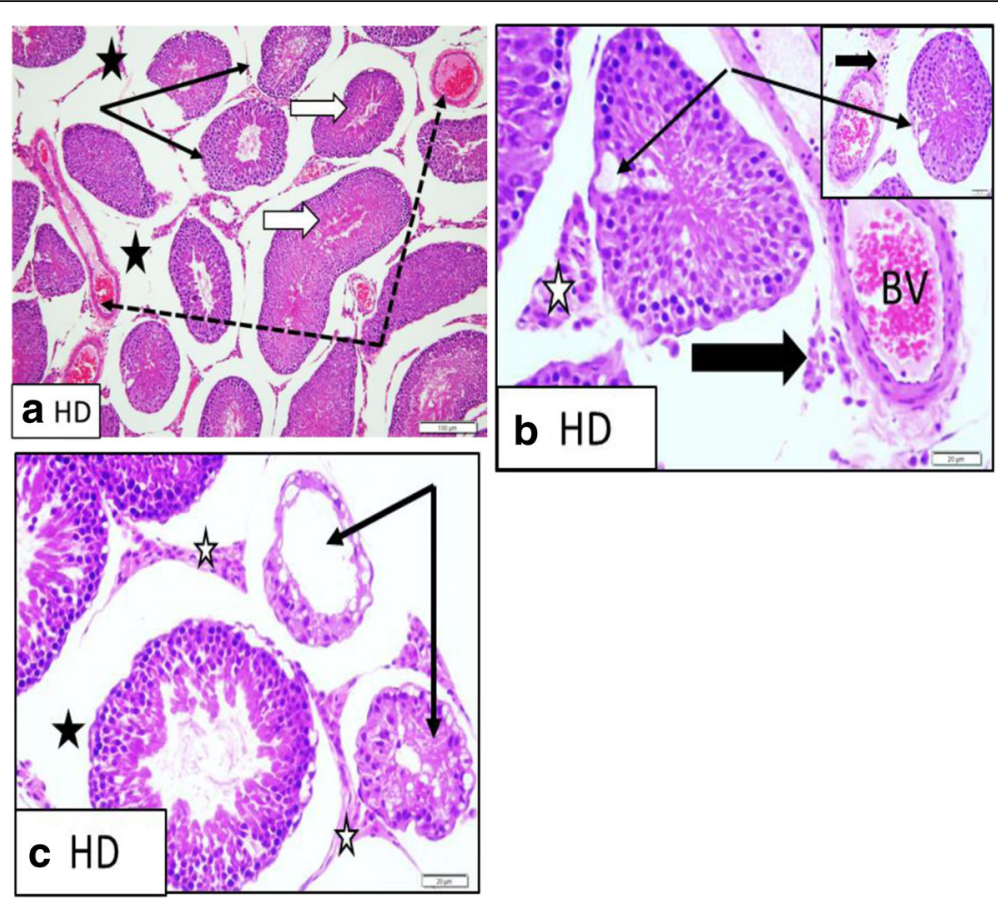

Fig. 7 Sections from the rat testis administered with high-dose hair dye for 8 days show the following: a more shrinkage (black arrows) and deformity (white arrows) of seminiferous tubule, wide interstitial spaces (black stars), and dilatation and congestion of blood vessels (dotted arrows) $(H-E, \times 200)$. b Magnified part to show degenerative changes in basal cells. The nuclei of other germ cells are dark stained and pyknotic (black arrows) marked congestion of blood vessels (BV) with perivascular inflammatory cells (thick black arrow) and atrophy and degeneration of Leydig cells (white star) $(\mathrm{H}-\mathrm{E}, \times 400)$. c Some tubules showed marked degeneration and absence of most germ cells (black arrows); the rest showed no mature sperms. Leydig cells looked small and degenerated (white and black stars) $(H-E, \times 400)$

One study reported that sections of treated liver showed mild chronic inflammations with one necroinflammatory focus, which with increasing the dose become multiple foci (Abd-ElZaher, Fawzy, Ahmed, Abd-Allah, \& Gayyed, 2012). Another study reported that the histopathological findings include centrilobular coagulative necrosis, periportal inflammation, fibrinous deposition, hemorrhages, and increased accumulation of neutrophils within hepatic parenchyma. The PPD-mediated hepatotoxicity seems to be enhanced by increased accumulation of neutrophils (Bharali \& Dutta, 2009). The histopathological findings in a different study included congestion of central and portal veins, with cellular infiltration; disruption of blood sinusoids and necrotic of Kupffer cells, as well as proliferation of bile ducts and focal necrosis of hepatic cells with deformed nuclei which appeared in pyknosis and karyolysis stages. Vacuolar cytoplasmic degeneration led to focal lysis of liver parenchyma which is showed in female rats that were administered with $0.001 \mathrm{ml}(1 \mathrm{mg} / \mathrm{kg} / \mathrm{bw})$ of PPD topically for 6 months (Alalwani, 2013). It is reported that the PPD induces free radicals and subsequently results in lipid peroxidation, which led to liver cell necrosis and renal tissue necrosis (Alalwani, 2013).

Current study also demonstrated the toxic effects of hair dye $\mathrm{X}$ on the rat kidney. Administration of low dose produced slight changes in proximal tubules in the form of increased cytoplasmic acidophilia and denser of nuclei staining (signs of early apoptosis). Distal tubules showed dilated lumina with decreased height of lining epithelium. Some tubular cells showed unstained cytoplasm. In the high dose of hair dye $\mathrm{X}$, the toxic effects showed disorganization of tubular shape, dilatation, and signs of cell degeneration with decreased nuclear size.

The present findings are in line with other studies which reported that hair dye is a potent nephrotoxic cocktail containing PPD, propylene glycol, and resorcinol and there was strong evidence of severe nephrotoxicity in humans and histopathological changes of acute tubular necrosis (Senthilkumaran \& Thirumalaikolundusubramania, 2015). The mechanisms of kidney injury following hair dye poisoning are many. The PPD itself has a direct toxic effect on the kidney due to its aromatic structure, which makes its easy reabsorption and concentration in tubule and can cause acute renal failure (ARF). Rhabdomyolysis is the main cause of ARF. Hypovolemia and metabolites of PPD also contribute to acute kidney injury (AKI) (Sanchez et al., 2016; Senthilkumaran \& Thirumalaikolundusubramania, 2015). Another study also reported marked peritubular capillary congestion in the cortex as well as medulla (Behera, Mridha, Kumar, \& Millo, 2015). 
In a study which analyzed 25 hair dye poisoning deaths, the histological examination of kidney cut section showed increased cellularity of glomeruli in $84 \%$ of the cases, swollen tubular lining cells with pale cytoplasm in $76 \%$ of the cases, and focal areas of total loss of tubular lining cells in $72 \%$ of the cases (Alugonda et al., 2013). Most of the histopathological changes appeared more pronounced on the proximal convoluted tubules (PCTs) than distal convoluted tubules (DCTs). PCTs at $3 \mathrm{mg} / \mathrm{kg}$ of PPDtreated group showed brush border fragments, cell necrosis, and nuclear pyknosis and karyolysis (Hummdi, 2012).

In this research, we also studied the effects of hair dye $\mathrm{X}$ on the rat testis. Section from the rat testis administered with low-dose hair dye for 8 days showed deformity and shrinkage of seminiferous tubules. Interstitial spaces were increased with compensatory hyperplasia of Lyedig cells. Sections from the rat testis administered with high-dose hair dye for 8 days showed more shrinkage and deformity of seminiferous tubule, wide interstitial spaces, and dilatation and congestion of blood vessels. However, other studies reported that hair dyes and their ingredients pose no risk of adverse reproductive effects and a series of oral reproductive toxicology or embryo-fetal toxicity studies on commercial hair dyes found no evidence for reproductive effects (Nohyneka et al., 2004). These findings do not agree with the results reported in the present study; this might be due to that commercial hair dyes may contain varying amounts of paraphenylenediamine and even undeclared chemical ingredients.

\section{Conclusions}

The hair dye poisoning is becoming a common suicidal poisoning. The current study showed that the commercial hair dye $\mathrm{X}$ at doses of 2.5 and $5 \mathrm{mg} / \mathrm{kg}$ orally produced histopathological changes and toxicity in the rat heart, liver, kidney, and testis. Therefore, it is important to raise the public awareness about the potential toxicity of the hair dyes.

\section{Abbreviations \\ AKI: Acute kidney injury; ARF: Acute renal failure; BD: Bile duct; CO: Control group; CV: Central vein region; DCTs: Distal convoluted tubules; EDTA: Ethylenediaminetetraacetic acid; HD: High-dose dye; H-E: Hematoxylin and eosin; KSA: Kingdom of Saudi Arabia; LD: Low dose; OECD: The Organization for Economic Co-operation and Development; PCTs: Proximal convoluted tubules; PPD: Paraphenylenediamine; PV: Portal vein; \\ ST: Seminiferous tubules}

\section{Acknowledgements}

We acknowledge the support of Northern Border University, KSA

\section{Funding}

The research is funded by a private partnership.

\section{Availability of data and materials}

The datasets generated and analyzed during the current study is available from the corresponding author on reasonable request.

\section{Authors' contributions}

TMA wrote the manuscript, contributed to the design of the study, and carried out the data analysis. KA coordinated the design of the study and was responsible for the execution of the experiment. MEM, AY, AY, RSH, and SDA helped to write the manuscript and contributed to the design of the study and to the literature review, and critically revised the manuscript. All authors read and approved the final manuscript.

\section{Ethics approval}

All animal experimentations were in agreement with the Ethical Principles for Animal Research adopted by Pharmacology and Toxicology, Faculty of Pharmacy, Northern Border University, Saudi Arabia.

\section{Competing interests}

The authors declare that they have no competing interests.

\section{Publisher's Note}

Springer Nature remains neutral with regard to jurisdictional claims in published maps and institutional affiliations.

\section{Author details}

'Department of Biological Sciences, Faculty of Science, Northern Border University, Arar, Saudi Arabia. ${ }^{2}$ Faculty of Pharmacy, Northern Border University, Rafha, Saudi Arabia. ${ }^{3}$ Department of Pharmacology and Toxicology, Faculty of Pharmacy, Northern Border University, Rafha, Saudi Arabia.

Received: 25 January 2018 Accepted: 9 April 2018

Published online: 24 April 2018

\section{References}

Abdel-Moneim, A. (2017). Acute toxicity by hair dye in upper Egypt. International Journal of Forensic Science \& Pathology, 5, 305-311.

Abdelraheem, M., El-Tigani, M., Hassan, E., Ali, M., Mohamed, I., \& Nazik, A. (2010). Paraphenylene diamine (hair dye) poisoning in children. Arab Journal of Nephrology and Transplantation., 3, 39-43.

Abd-ElZaher, M.., Fawzy, I., Ahmed, H., Abd-Allah, A., \& Gayyed, M. (2012). Some toxicological health hazards associated with subchronic dermal exposure to paraphenylene-diamine (PPD): An experimental study. Egyptian Journal of Basic and Applied Sciences., 2, 105-111.

Alalwani, A. (2013). Histopathological examination of paraphenylen diamine toxicity in female rats liver. Engineering Science and Technology., 3, 296-302.

Alghamdi, K., \& Moussa, N. (2012). Local side effects caused by hair dye use in females: Cross-sectional survey. Journal of Cutaneous Medicine and Surgery. $16,39-44$.

Al-Shaikh, T. (2016). The protective role of captopril against cardiac toxicity of different doses of paracetamol: Histological study in rats. Journal of the North for Basic and Applied Sciences, 1(2), 119-124.

Alugonda, Y., Maddileti, B., Naga Lingam, J., \& Rangaiah, Y. (2013). A medico-legal study of hair dye poisoning. Journal of Indian Academy of Forensic Medicine., $35,251-254$

Behera, C., Mridha, A., Kumar, R., \& Millo, T. (2015). Characteristic autopsy findings in hair dye poisoning. BMJ Case Reports., 2015, 1-2.

Bharali, M., \& Dutta, K. (2009). Hepatic histopathological abnormalities in rats treated topically with para-phenylene diamine (PPD). Journal of Pharmacology and Toxicology, 4, 221-228.

Bhargava, P., \& Matthew, P. (2007). Hair dye poisoning. Journal of the Association of Physicians of India., 55, 871-872.

Chandran, J., Manners, R., Agarwal, I., \& Ebenezer, K. (2012). Hair dye poisoning in a paediatric patient. Case Reports in Pediatrics., 2012, 1-3.

Chua-Gocheco, A., Bozzo, P., \& Einarson, A. (2008). Safety of hair products during pregnancy. Canadian Family Physician., 54, 1386-1388.

Garg, K., Tiwari, R., \& Ahlawat, A. (2014). Hair dye poisoning: An unusual encounter. Indian Journal of Critical Care Medicine., 18, 402-404.

Hassan A-Rahman, N., \& Ahmed, A. (2014). Hair dye poisoning: An overview. Journal of Scientific Research., 2, 31-33.

Hummdi, L. (2012). Histopathological alterations in renal tubules of female rats topically treated with paraphenylen diamine. World Applied Sciences Journal, $16,376-388$

Jain, P., Agarwal, N., Kumar, P., Sengar, N., Agarwal, N., \& Akhtar, A. (2011). Hair dye poisoning in Bundelkhand region (prospective analysis of hair dye 
poisoning cases presented in Department of Medicine, MLB Medical College, Jhansi). Journal of the Association of Physicians of India., 59, 415-419.

Kondle, R., Pathapati, R., Saginela, S., Malliboina, S., \& Makineedi, V. (2012). Clinical profile and outcomes of hair dye poisoning in a teaching Hospital in Nellore. International Scholarly Research Notices., 2012, 1-5.

Nohyneka, G., Fautzb, R., Benech-Kiefferc, F., \& Toutaina, H. (2004). Toxicity and human health risk of hair dyes. Food and Chemical Toxicology., 42, 517-543.

Prabhakar, Y., \& Kamalakar, K. (2012). Hair dye poisoning: A report of three cases. Journal Dr.NTR University of Health Sciences, 1, 46-48.

Punjan, N. (2014). Paraphenylene diamine (hair dye) poisoning leading to critical illness neuropathy. Neurological Disorders., 2, 2-5.

Saitta, P., Cook, C., Messina, J., Brancaccio, R., Wu, B., Grekin, S., \& Holland, J. (2013). Epidemiological evidence relating personal hair dye use to the risk of malignancy. Journal of Clinical and Aesthetic Dermatology, 6, 39-46.

Sakuntala, P., Musa Khan, P., Sudarsi, B., Manohar, S., Siddeswari, R., \& Swaroop, K. (2015). Clinical profile and complications of hair dye poisoning. International Journal of Science and Research., 5, 1-4.

Sanchez, L., Handyal, H., Kannan, S., Siddalingeshwara, Alvarez-Uria, G., Gavalda, L., \& Corbella, X. (2016). Hair dye poisoning: Retrospective analyses of patients admitted to ICU at a rural hospital in India. Indian Journal of Medical Research, 144, 134-137.

Senthilkumaran, S., \& Thirumalaikolundusubramania, P. (2015). Acute hair dye poisoning: Lurking dangers. Journal of Mahatma Gandhi Institute of Medical Sciences., 20, 33-37.

Tiwari, D., Jatav, O., \& Dudani, M. (2016). Prospective study of clinical profile in hair dye poisoning (PPD) with special reference to electrocardiographic manifestations. International Journal of Medical Science and Public Health., 5, 1313-1316.

Zaid, A., Al- ramahi, R., Abu ghoush, A., Jaradat, N., \& Musmar, M. (2013). Frequency and attitudes of using hair dyes among Palestinian women International Journal of Pharmaceutics, 5, 485-488.

\section{Submit your manuscript to a SpringerOpen ${ }^{\circ}$ journal and benefit from:}

- Convenient online submission

- Rigorous peer review

- Open access: articles freely available online

- High visibility within the field

- Retaining the copyright to your article

Submit your next manuscript at $\boldsymbol{s p r i n g e r o p e n . c o m ~}$ 